Chimia 46 (1992) 262-267

(C) Neue Schweizerische Chemische Gesellschaft ISSN 0009-4293

\title{
Einführung und Motivation von und für TQM in der Verfahrensentwicklung für Feinchemikalien
}

\author{
Hans-Rudolf Dettwiler*
}

Es ist uns allen bewusst, dass wir in einer Zeit der überstürzten Beschleunigung des wissenschaftlichen und technischen Fortschrittes leben. Der starke Konkurrenzdruck, gepaart mit neuen technologischen Möglichkeiten sowie mit rasch sich ändernden Anforderungen von Markt (Stichworte EWR, EG), Umfeld und Gesellschaft (Stichwort Umbruch in Osteuropa), stellt zunehmend höhere Anforderungen an die Innovationsfähigkeiten eines Unternehmens. Es wird notwendig, noch schneller auf völlig neue Technologien, sich neu bildende Kunden- und Marktsegmente und ein sich änderndes Marktumfeld einzugehen. Für uns als produzierende Industrie wird diese Entwicklung begleitet durch die Elemente Kosten und Konkurrenzsituation. Im Letzteren verbirgt sich bereits das Kriterium der Qualität, welches das Thema dieses Beitrages darstellt. Im Gegensatz zur Hochschulforschung mit ihrer Ausrichtung auf die Basis-Ideen und Erkenntnisse, muss die industrielle Forschung ihr Augenmerk auf die Produkte und ihre Anwendung richten. Sie beeinflusst dadurch in einem frühen Zeitpunkt das Qualitätsumfeld neuer Produkte. Die industrielle F+E stellt heute ein Netz integrierter Aktivitäten dar, vom Entstehen einer Idee bis zu deren Verwirklichung und Anwendung. In Abstimmung mit dem Management muss die F+E-Leitung bestrebt sein, die lückenlose Integration der Forschung in das Unter-

\footnotetext{
*Korrespondenz: Dr. H.-R. Dettwiler Entwicklungsabteilung

Lonza $A G$

CH-3930 Visp
}

nehmen sicherzustellen. Mit der Einführung eines neuen Qualitätssicherungssystems stellen sich für $\mathrm{F}+\mathrm{E}$ die Fragen der konzeptionellen und organisatorischen Gestaltung und des ‘Verkaufens' der Qualitätspolitik an die Mitarbeiter in F+E.

Dies leitet über zur Frage, wie die F+EMitarbeiter für die Belange der Qualität interessiert werden können. Die Motivation des Forschers und Entwicklers in der Industrie ergibt sich aus der Zielsetzung des Erreichens einer guten Innovation. Als Innovation bezeichnen wir die Umwandlung des wissenschaftlichen und technologischen Fortschritts in der Chemie und der chemischen Technologie in greifbare Ergebnisse (Produkte). Diese müssen dem Verbraucher einen substantiellen Vorteil und dem Hersteller einen vernünftigen Gewinn bringen. Eine Entwicklung kann letztlich nur als innovativ bezeichnet werden, wenn sie die Bedürfnisse des Käufers und damit letztlich der Gesellschaft auf einem hohen qualitativen $\mathrm{Ni}$ veau zu befriedigen vermag.

Die Geschäftseinheit LONZA ORGANICS innerhalb der Alusuisse-Lonza Holding steht heute als einer der bedeutenden Feinchemikalienhersteller im Chemiegeschäft. Zum besseren Verständnis der qualitätsrelevanten Ausführungen sollen vorerst Merkmale dieses Geschäftsbereiches kurz skizziert werden.

\section{LONZA ORGANICS im Feinchemie- geschäft und Marktumfeld}

Bei der organischen Chemie und den Feinchemikalien verfügen wir bei $L O N$ $Z A$ über eine vorzügliche, ausbaufähige Ausgangslage. Hier ist eine vertikal integrierte, gut funktionierende Struktur vorhanden, die die Umwandlung von Roh-

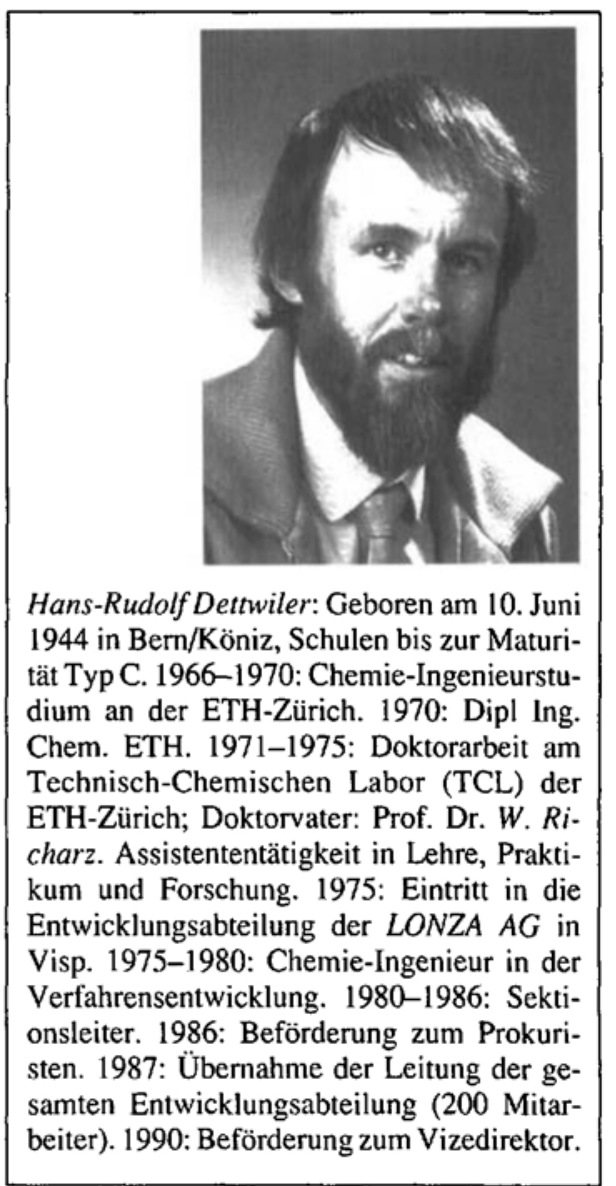

stoffen zu Grundchemikalien bis hin zu Produkten mit hoher Wertschöpfung abdeckt. Zu unseren ausgeprägten Stärken zählen Forschung und Entwicklung. Ausserdem ist unser Produkt- und Technologie-Portfolio breit angelegt und wir sind in der Lage, die ökologischen Rahmenbedingungen einzuhalten.

Die Produktepalette im Bereich der organischen Chemie lässt sich einteilen in:

Basis-Feinchemikalien charakterisiert durch

- Am Markt bekannte und eingeführte chemische Produkte

Spezial-Feinchemikalien charakterisiert durch

- Neue von den Verbrauchern (Industrie) gesuchte allgemein verwendbare Feinchemikalien

- Neue Zwischen- und Endprodukte für auf dem Markt noch nicht eingeführte Spezialitäten. Diese Produkte werden in der Regel in einer engen, vertraglich abgesicherten Zusammenarbeit zwischen dem Kunden und LONZA ORGANICS von der Laborstufe bis zu einer industriellen Produktion entwikkelt.

Die Haupttätigkeit von $L O N Z A O R$ GANICS ist die Herstellung hochwertiger organisch-chemischer Zwischenprodukte und Wirkstoffe für die

- Pharma-Industrie

- Pflanzenschutzmittel-Industrie 
- Farbstoff- und Pigmentindustrie

- Kunststoff-Industrie

Zu bemerken ist, dass keine formulierten Endprodukte hergestellt werden.

\subsection{Aufgaben und Zielsetzungen von $F+E$ innerhalb LONZA ORGANICS}

Aus den obgenannten Zielgebieten für den Geschäftsbereich lassen sich Aufgaben und $Z$ ielsetzungen von $F+E$ innerhalb LONZA ORGANICS ableiten. Die in Visp lokalisierte Forschung und Entwicklung wird heute einerseits für die Suche neuer Verfahren und Technologien für bestehende Produkte und anderseits zur Weiterentwicklung der Produktegruppe Spezial-Feinchemikalien für die obgenannten Produktbereiche eingesetzt. Unsere Tätigkeit ist daher weitgehend auf konkrete Bedürfnisse des Marktes ausgerichtet. Entsprechend lassen sich die Hauptaufgaben für $\mathrm{F}+\mathrm{E}$ wie folgt formulieren:

- Suche nach neuen synthetischen Wegen.

- Entwicklung von chemischen Verfahren zur Herstellung von Feinchemikalien von der Idee bis zur Produktionsreife.

- Mit minimalem Aufwand an Zeit und Kosten für die Produkte- und Verfahrensentwicklung.

- MitdemSicherstellen derökologischen Verträglichkeit und der Erarbeitung eines Entsorgungskonzeptes.

- Mit der umfassenden Bearbeitung und Beurteilung der Sicherheitsaspekte.

- Mit der Entwicklung eines validierten Verfahrens bezüglich Chemie, Verfahrenstechnik und Parameterempfindlichkeit.

- Mit der Gewährleistung der Einhaltung von Spezifikationen neuer Produkte in klar vorgegebenen Bereichen.

Mit dem Ziel:

- Das Wachstum sicherzustellen

- Die Ertragskraft zu verbessern.

Es versteht sich von selbst, dass wir nur mit der entsprechenden Qualität unserer Produkte und Dienstleistungen für unsere Kunden ein verlässlicher Partner sein und bleiben werden. Die Entwicklung des entsprechenden Qualitätsbewusstseins auf allen Stufen und speziell bereits auf Stufe $\mathrm{F}+\mathrm{E}$ ist daher für das Gedeihen des Unternehmens unerlässlich. Kundenbedürfnisse müssen verstanden werden, um die mit dem Kunden vereinbarten Leistungen und Lieferungen fehlerfrei zu erfüllen. Unser Kunde will genau das spezifizierte Produkt und ist immer mehr aus Gründen der Registrierung auf entsprechend autorisierte und verlässliche Partner angewiesen.

Tab. 1. Strukturierung der Normen für die F+E Tätigkeit in der Chemie

\begin{tabular}{|c|c|c|c|}
\hline \multicolumn{2}{|c|}{ Forderungen an F+E ais Kap. 4 ISO 9001} & \multicolumn{2}{|l|}{ Regelung } \\
\hline & & für & in \\
\hline 4.4 & Design-Lenkung & \multirow{5}{*}{ Organisation } & \multirow{5}{*}{ Q-Manual } \\
\hline 4.4 .1 & Allgemeines & & \\
\hline 4.4 .2 & Design- und Entwiclungsplanung & & \\
\hline 4.4 .2 .1 & Zuordungn der Tätigkeiten & & \\
\hline 4.4 .2 .2 & Organisatorische und technische Schnittstellen & & \\
\hline 4.4 .3 & Design-Vorgaben & \multirow{4}{*}{$\begin{array}{l}\text { F+E-Ablauf } \\
\text { (Projektablauf) } \\
\text { inkl. Ökologie, } \\
\text { Sicherheit }\end{array}$} & \multirow[t]{2}{*}{ QS-RL 4.1} \\
\hline 4.4 .4 & Design-Ergebnis & & \\
\hline 4.4 .5 & Design-Verifizierung & & \multirow[t]{2}{*}{ QS-RL 4.3} \\
\hline 4.4 .6 & Design-Änderungen & & \\
\hline 4.5 & Lenkung der Dokumente & \multirow{3}{*}{ Dokumentation } & \multirow{3}{*}{ QS-RL 4.2} \\
\hline 4.5 .1 & Genehmigung und Herausgabe von Dokumenten & & \\
\hline 4.5 .2 & Änderung/Modifikation von Dokumenten & & \\
\hline
\end{tabular}

\section{Aufbau des Qualitätssicherungs- Systems bei LONZA ORGANICS}

Wir erstehen das TQM primär als eine Qualitätsphilosophie und als einen anzustrebenden Idealzustand in der laufenden Verbesserung unserer industriellen Leistungen. Mit derEinführung der ISO-Normen und dem Erreichen der entsprechenden Zertifizierung legen wir einen ersten Grundstein für den Aufbau von TQM. Das Weiterarbeiten am System bedeutet für alle Bereiche eine permanente Aufgabe basierend auf klaren Grundsätzen undZielsetzungen.

Aus den Grundsätzen der Qualitätspolitik und der Q-Sicherung ergeben sich folgende für die $F+E$ relevanten Elemente:

- Die unternehmerische Verantwortung für eine Q-Politik liegt bei der Geschäftsleitung. Diese kann sich der obersten Verantwortung nicht entziehen. Sie muss mit aktivem Engagement vorangehen.

- Anderseits ist jede Mitarbeiterin und jeder Mitarbeiter verpflichtet ihren/seinen persönlichen Beitrag für die Qualität zu leisten. Bewusste Auseinandersetzung und Identifikation mit den Regeln der Q-Sicherung wird gefordert.

- Die Qualitätsschulung und Motivation der Mitarbeiter stellen zwei zentrale Elemente zur Durchsetzung eines Qualitätssicherungssystems dar.

Insbesondere die Q-Schulung wirdeine permanente Aufgabe darstellen, müssen doch Neueintretende (was in einer F+E häufig der Fall ist) in das System eingeführt und die Mitarbeiter laufend bezüglich Q-relevanten Änderungen ausgebildet werden. Nicht zu unterschätzen ist, dass bei einer Neueinführung Probleme und Fallstricke zu überwinden sind, auf die wir im Kapitel 'Motivation' noch näher eingehen werden.

\subsection{Das Q-Handbuch von LONZA ORGANICS}

Die Geschäftseinheit hat ihr Qualitätshandbuch erarbeitet. Dieses bildet quasi die 'Q-Verfassung' von LONZA ORGANICS. Es orientiert sich im Aufbau an der Norm SN EN 29 001/ISO 9001. Darin enthalten sind $20 \mathrm{Kapitel}$, wovon sich Kap. 4 ausschliesslich mit der Qualitätssicherung in Forschung und Entwicklung (Design-Lenkung) beschäftigt. Auf den $\mathrm{Ge}-$ samtinhalt des Handbuches wird im Weiteren nicht eingegangen. Wir werden uns spezifisch mit den für $\mathrm{F}+\mathrm{E}$ relevanten Aspekten auseinandersetzen.

\subsection{Forderungen des QS-Systems an $F+E$}

Um aufzuzeigen wie sich ein QS-Systems auf eine $\mathrm{F}+\mathrm{E}$ auszuwirken vermag, wird nachfolgend auf die ISO-Normen 9001/9004 und das Kapitel Design-Lenkung (Kap. 4.4) ausführlich eingegangen.

Es gilt nun zu beachten, dass die Begriffe der ISO-Norm aus der Maschinenindustrie stammen und man vor der Situation steht, die Normen auf die Gegebenheiten der chemischen F+E zu 'übersetzen'. Zusätzlich spielt die Dokumentation von $\mathrm{F}+\mathrm{E}$ Ergebnissen in der chemischen Indu- 
strie eine zentrale Rolle. Daher wird auch das entsprechende Kapitel der ISO-Normen für den $\mathrm{F}+\mathrm{E}$-Ablauf einbezogen werden müssen.

Aus Tab. 1 ist die entsprechende Kapitelnumerierung der ISO-Norm ersichtlich und wie die Interpretation für die Strukturierung der $\mathrm{F}+\mathrm{E}$ Tätigkeit erfolgt ist.

Aus der Analyse der Normen des Kap. 4 glauben wir nebst einer klaren Festlegung der Organisation mit drei Qualitätssicherungsrichtlinien für den Bereich $\mathrm{F}+\mathrm{E}$ auszukommen. Diese beinhalten:

den Projektablauf die Dokumentation die Sicherheit

QS-Richtlinie 4.1 QS-Richtlinie 4.2 QS-Richtlinie 4.3

Damit meinen wir den verlangten Anforderungen gerecht zu werden. Nachfolgend wird auf den Inhalt dieser QS-RL eingegangen.

\subsection{Qualitätssicherungsrichtlinien für den Bereich $F+E$}

Die F+E-Organisation ist eingebettet in die Linienorganisation der Geschäftseinheit $L O N Z A$ ORGANICS, die ihrerseits wieder ein Element der übergeordneten Bereichsstruktur der Alusuisse-Lonza Holding darstellt. Diese Organisationsstruktur ist im Q-Manual für LONZA ORGANICS abgedeckt. Wir werden auf dieses nicht weiter eingehen, sondern uns spezifisch den Gegebenheiten, wie sie in den QS-Richtlinien 4.1-4.3 geregelt werden, zuwenden.

Tab. 2. Unterschied Maschinen/Chemische Industrie bezüglich Auslegung der Normen im Projektablauf

\begin{tabular}{|c|c|c|}
\hline Norm & Maschinenindustrie & Chemische Industrie \\
\hline Design-Vorgabe & $\begin{array}{l}\text { Beschreibung der } \\
\text { Anforderungen } \\
\text { Pflichtenheft }\end{array}$ & $\begin{array}{l}\text { Evaluationsphase } \\
\text { Vorevaluation, Evaluation, } \\
\text { Chemische Struktur, Spezifikation, } \\
\text { Preis, Timing }\end{array}$ \\
\hline Design-Ergebnis & $\begin{array}{l}\text { Konstruktionspläne } \\
\text { Prototyp }\end{array}$ & $\begin{array}{l}\text { Verfahrensforschung/ } \\
\text { Verfahrensentwicklung } \\
\text { Literatur, Synthesestrategie. } \\
\text { Laborverfahren }\end{array}$ \\
\hline Design-Verifizierung & $\begin{array}{l}\text { Nullserie, Überprüfung der } \\
\text { Erfüllung des Pflichtenheftes }\end{array}$ & $\begin{array}{l}\text { Pilotierungsphase } \\
\text { Verfahrensentwicklung } \\
\text { Pilotanlage, Versuchsfabrikation }\end{array}$ \\
\hline Produktionsverfahren & Produktionsverfahren & $\begin{array}{l}\text { Produktionsphase } \\
\text { Produktionsverfahren }\end{array}$ \\
\hline
\end{tabular}

auf bei bein festgelegt wird, der die Erfüllung der Anforderungen (Spezifikationen, Ausbeute, Wirtschaftlichkeit, Termine) ermöglicht. Dieser Anforderung kann nach unserer Meinung nur mit der Implementierung eines konsequenten Projektmanagements nachgelebt werden, das die spezifischen Bedürfnisse von $\mathrm{F}+\mathrm{E}$ und die Forderungen der ISO-Normen in sich vereinigt. Da sehr viele unterschiedliche Fachbereiche unter einen Hut gebracht werden müssen, wird die Bereitschaft zur interdisziplinären Zusammenarbeit eine wichtige Rolle spielen. Der Information und Kommunikation auf allen Ebenen kommt zentrale Bedeutung zu. Der Auswahl und der Einsetzung von Projekteams muss grosse Beachtung geschenkt werden. Nebst den organisatorischen Schnittstellen nach innen und nach aussen werden die Tätigkeiten mittels Pflichtenheften und individuelle Festlegung der wichtigsten Zuständigkeiten (WIZU) festgelegt.

Es muss sichergestellt werden, dass alle Stellen, die in der Entwicklung eines Produktes involviert sind, sich der Verantwortung zum Erreichen des Qualitätszieles bewusst sind. Dies ist nun vor allem auch ein Thema der Schulung und Motivation auf allen Stufen.

Das Festlegen eines definierten Projektablaufes von der Evaluation bis zum Abschluss der Verfahrensentwicklung ist eine Vorgehensweise, die den Bedürfnissen von F+E, Kunden und ISO-Normen umfassend gerecht werden kann.

\subsubsection{Das Projektablaufschema und die Projektablaufphasen}

Um die in der Norm vorgegebenen Schritte für die Tätigkeit einer $F+E$ in der chemischen Industrie zu interpretieren, muss man sich, wie vorgangs erwähnt, vergegenwärtigen, wie in der Maschinenindustrie die Entwicklung von neuen Apparaten/Geräten abläuft und wie dieses Vorgehen sich im Vergleich zur chemischen Industrie präsentiert. In $T a b .2$ ist ersichtlich, welche Projektablaufphasen wir für unsere $\mathrm{F}+\mathrm{E}$ für wichtig halten.

Die ISO-Norm verlangt, dass die Arbeiten in F+E geplant werden. Dies wird durch diesen normierten Ablauf gewährleistet, der den in Tab. 2 definierten Phasen (Evaluation, Chemische Verfahrensforschung/Verfahrensentwicklung, Pilotierung, Produktion) Rechnung trägt.

Lassen Sie uns die einzelnen Phasen kurz bezüglich ihrer wesentlichen Elemente anhand der Graphik in der Figur analysieren.

\subsubsection{Projektphase 1: Evaluation}

a) Die Vorevaluation

Die Normen verpflichten die Marktentwicklung, den Marktbedarf für ein neues Produkt festzulegen, seine Spezifikationen in einem Pflichtenheft (Projektidee, product brief) festzulegen und die tatsächliche Situation beim Kunden festzuhalten (Projektidee).

Die Marktentwicklung wird verpflichtet, sich qualitätsbezogene Informationen zu beschaffen, mit den Kundenerwartungen zu vergleichen und dem Kunden eine geeignete Rückinformation zukommen zu 


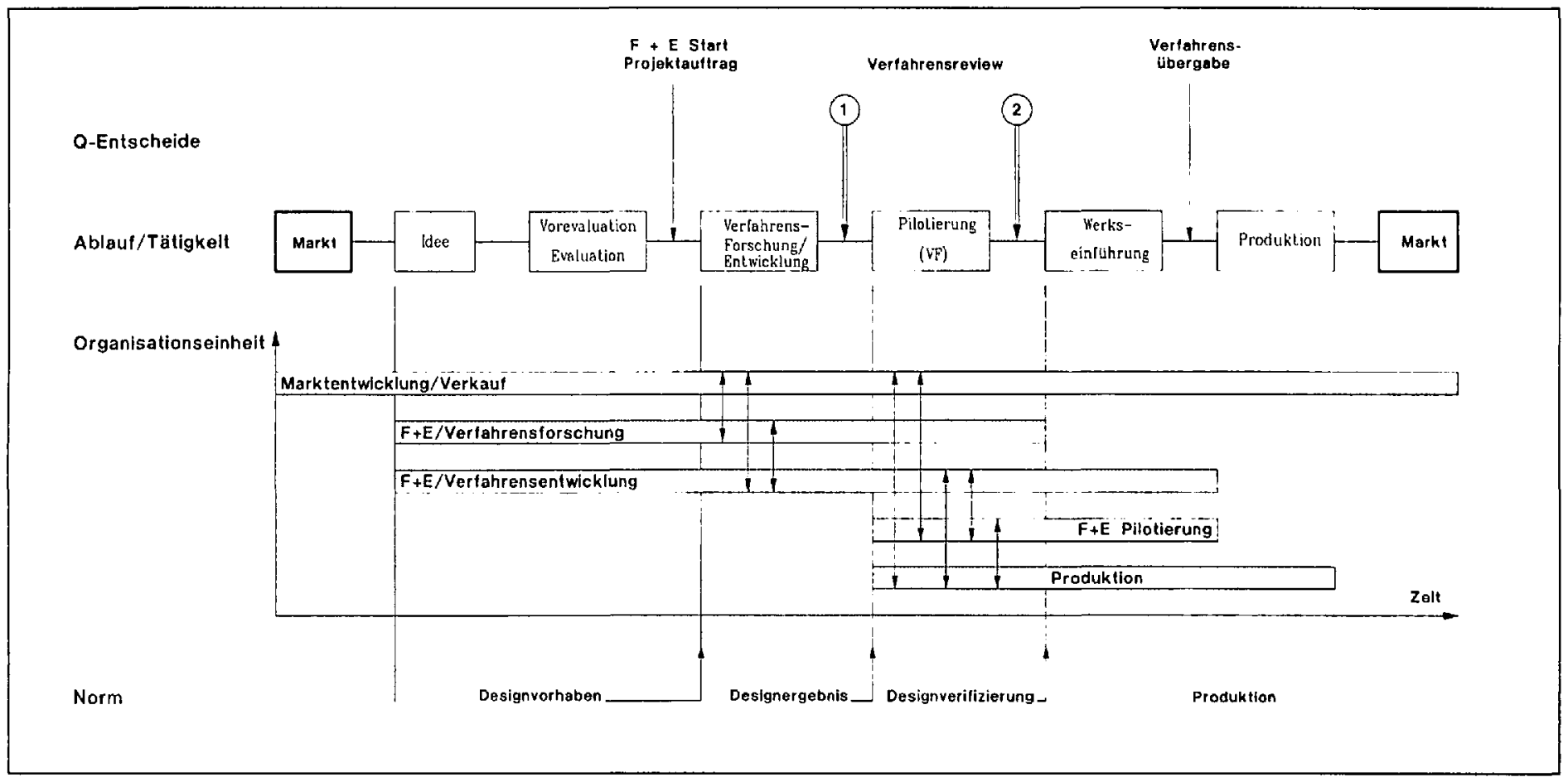

Figur. Die Prokektablaufphasen für $F+E$

lassen (Kontinuierliches 'feed-back' System zum Kunden).

$\mathrm{F}+\mathrm{E}$ muss klare und technisch eindeutige Daten über die Anforderungen (Spezifikationen, Preisgestaltung, Wirtschaftlichkeit, Timing) erhalten. Insbesondere müssen die Produktspezifikationen eindeutig festgelegt sein, sodass sich klare Annahme bzw. Rückweisungskriterien aufstellen lassen.

\section{b) Die Evaluation}

Von $\mathrm{F}+\mathrm{E}$ verlangen die Normen, dass die von der Marktentwicklung formulierten Vorgaben bezüglich Chemisch/Technische Machbarkeit, Wirtschaftlichkeit und Realisierbarkeit eines beherrschbaren Verfahrens überprüft werden. Die F+E muss in zeitliche Phasen gegliederte Entwicklungsprogramme festlegen, deren Umfang der Komplexizität der Aufgabe angepasst ist. Der erwartete F+E-Aufwand muss bezüglich Kosten und Zeitplan quantifiziert werden. Das Verbesserungspotential und die mögliche Eigenleistung ist offenzulegen und zu begründen. Der mit der technischen Evaluation betraute Forschungs- und Entwicklungschemiker/Ingenieur muss auch zwingend Anforderungen bezüglich Umwelt, Sicherheit sowie andere rechtliche Vorschriften beachten.

Am Ende der Evaluationsphase steht ein Entscheid für oder gegen die Aufnahme eines F+E Projektes. Dieser Entscheid beruht grundsätzlich auf einer Konsensfindung zwischen Marktentwicklung und F+E. Ein positiver Entscheid führt zur Auslösung einesF+E-Projektauftrages, der nach LONZA-eigenen integralen Projektmanagementgrundsätzen abgewickelt wird und der die Einsetzung eines Projektleiters bereits im Anfangstadium der F+ETätigkeit zur Folge hat.

Die ISO-Norm verlangt, dass Forschungs- und Entwicklungstätigkeiten qualifiziertem Personal übertragen wird. Dies beinhaltet auch Ausbildungsmassnahmen (neben dem primären Ausbildungsprogramm der Personalabteilung), die durch $\mathrm{F}+\mathrm{E}$ direkt erfolgen mussen wie:

- die abteilungsbezogene Grundschulung neueingestellten Personals.

- Ermittlung von projektspezifischen chemischen/technischen Ausbildungsbedürfnissen.

- Schulung für Probenahme und Durchführung analytischer Prüfungen.

- Die Übertragung spezialisierter Aufgaben an Personal, das die nötige Erfahrung und nachgewiesene Fähigkeiten besitzt.

\subsubsection{Projektphase 2: Verfahrensfor-} schung/Entwicklung (Labor)

In enger Teamzusammenarbeit zwischen dem Forschungs-und Entwicklungschemiker wird die Verfahrensforschung/ Entwicklung auf Stufe Labor eingeleitet. Hierbei bearbeitet dererstere schwerpunktmässig die Chemie und der zweite die Verfahrenstechnik. Zielsetzung ist das Finden der chemisch optimalen Synthese, der technischen Machbarkeit und der wirtschaflichen Konkurrenzfähigkeit. Bereits hier wird dem zukünftigen Risikopotential und der Sicherheit des Verfahrens hohe Beachtung geschenkt. Das Entsorgungskonzept ist bezüglich der Schwerpunkte der zu erwartenden Probleme festzulegen.

\subsubsection{Projektphase 3: Pilotierung mitl} ohne Versuchsfabrikation

Die verfahrenstechnische Optimierung hat primär zur Zielsetzung, die Resultate des Labors im Technikumsmassstab zu bestätigen und die zu validierenden Verfahrensparameter abschliessend festzulegen.

Die sehr oft parallel zur Pilotierung ablaufende Versuchsfabrikation stellt die Belieferung der Kunden mit ersten grösseren Mengen sicher. Die Basis für die Produktfreigabe bildet das Erreichen der Zielwerte der Spezifikationen.

Der Abschluss der Verfahrensentwicklung mündet in das Erstellen einer definierten Projektdokumentation, die ihrerseits wieder die Basis für das Erstellen der Einfahrunterlagen im Werk bildet.

Dem verantwortlichen Projektleiter und seinem Team sind Hilfsmittel in die Hand zu geben, die seine Aufgabe erleichtern und repetitive Projektabläufe nach genormten Unterlagen ermöglichen. (Checkliste Projektablauf Entwicklung, Handbücher für sichere Laborarbeit und Pilotierung.)

Das Ziel der gesamten $F+E$-Tätigkeit ist gemäss ISO-Norm ein produktionsreifes validiertes und beherrschtes Verfahren sowie der Nachweis der Beherrschung.

\subsubsection{Projektphase 4: Werkseinfüh- rung und Produktion}

Auf die Werkseinführung neuer Verfahren kann hier nicht näher eingegangen werden. Es sei nur festgehalten, dass auch diese Projektphase klar strukturiert ist und der F+E-Projektleiter mit seinem Team 
(Know-How-Träger) in eine übergeordnete Projektorganisation eingebaut wird.

\subsubsection{Projektkontrolle}

Als Design-Ergebnis sehen wir, wie oben dargelegt, ein an die Fabrikation übergabereifes Verfahren an, das eine reproduzierbare Herstellung eines Stoffes bzgl. Ausbeute und Qualität unter wirtschaftlich vertretbaren Bedingungen erlaubt. Ein solches Verfahren muss dokumentiert werden und es muss nachgewiesen werden, dass es die Vorgaben erfüllt. Es müssen Annahmekriterien bzgl. der Produktqualität festgelegt sein. Die Reaktionsparameter, die für Sicherheit, Ausbeute und Produktqualität relevant sind, müssen systematisch dokumentarisch erfasst sein. Es kommen noch weitere Überprüfungen in Betracht wie:

- Use-Tests mit Labormustern beim Kunden (Funktionseignung)

- Vergleich mit Konkurrenzprodukten.

Die Projektkontrolle wird gewährleistet durch folgende organisatorische Instrumentarien:

- Periodische Sitzungen von F+E mit der Marktentwicklung für die Festlegung von kurzfristigen Projektkorrekturen

- F+E Sitzungen (zweimal jährlich) mit der Geschäftsleitung zur Festlegung strategischer Zielsetzungen.

- Als Qualitätsbezogene Kontrollmassnahmen stehen zur Verfügung:

- Die technische Beurteilung im Rahmen des Projektteams und als neu einzuführender Meilenstein

- Der Verfahrensreview (s. Fig.)

Die Norm 9001 verlangt, dass zum Abschluss jeder Design-Phase (Projektphasen 2 und 3) eine formelle, dokumentierte, systematische und kritische Überprüfung des chemischen Verfahrens vorgenommen wird.

(Design review, Für LONZA: Verfahrens-Review)

Folgende Hilfsmittel/Entscheidungsgrundlagen werden in den Entscheidungsgremien eingesetzt:

- Monatsberichte

- Projektstand (technischer Stand)

- Projektprofil (kommerzieller Stand inkl. Umsatzerwartung)

- Formular Verfahrens-Review

Der Verfahrens-Review muss von periodischen Projektsitzungen unterschieden werden. An diesen Überprüfungen sollten alle für die Qualität des Gesamtprojektes zuständigen Stellen teilnehmen. Probleme und Unzulänglichkeiten eines chemischen Verfahrens müssen anhand einer definierten Checkliste identifiziert werden. Weiter wird verlangt:
- Vergleich der Spezifikationen mit dem Anforderungsprofil (product brief).

- Vergleich mit Konkurrenzverfahren.

- Durchführbarkeit in bestehenden Anlagen.

- Vergleich mit ähnlichen, früher durchgeführten Verfahren.

\subsubsection{QS-RL 4.2. Erfassung, Dokumen-}

tation und Archivierung von $F+E$ -

Ergebnissen

Für die Dokumentation stehen bei uns

folgende Hilfsmittel im

Einsatz:

Elektronische Datenbanken:

- TRIP: Text-Retrieval-System für die Dokumentation von Forschungsberichten, Aktennotizen, Gestehungskostenschätzungen, Protokollen

- DARC: Strukturdatenbank

- ORAC: Reaktionsdatenbank

- TWR: Datenbanken für technisch-wissenschaftliches Rechnen

Dokumente

- Laborjournale

- Forschungsberichte (standardisiert)

- Projektordner (standardisiert)

\subsubsection{QS-RL 4.3. Sicherheit in For-} schung und Entwicklung

Da der Sicherheit chemischer Verfahren in Bezug ihrer Auswirkungen auf Mensch und Umwelt heute eine überragende Bedeutung zukommt, wurde es als angemessen angesehen für diesen Bereich eine eigene QS-RL zu schaffen.

Wesentliche Elemente dieser Richtlinien sind:

- Das Sicherheitsteam F+E. Es schlägt die für $F+E$ spezifischen Erlasse vor und steht mit dem Werkschutz in Verbindung.

- Das Handbuch für sicheres Arbeiten im Labor.

- Das Handbuch für sichere Pilotierung.

Die Risikoanalyse für Pilot- und Versuchsfabrikationsanlagen.

- Die Sicherheitsschulung.

\section{Motivation}

Die Einführung von Qualitätssicherungssystemen bewirkt in jedem Unternehmen einen bedeutenden Einschnitt in die Firmenkultur. Diese ist geprägt durch die Menschen, die Mitarbeiter und ihr persönliches Verhalten. Ab den 80er Jahren wurde erkannt, dass im Bereich der Qualität nicht nur die Produktqualität, sondern auch die Dienstleistung und vor allem das individuelle menschliche Verhalten für ein integrales Qualitätssicherungssystem von grundlegender Bedeutung ist. Unsere eigene persönliche Qualität ist die
Voraussetzung für jede andere Qualität. Es liegt auf der Hand, dass die Motivation eines jeden einzelnen für ein QS-System ein wesentlicher, wenn nicht entscheidender Faktor dafür darstellt, ob das System schlussendlich erfolgreich funktionieren kann.

In den Normen ist der Bereich der Motivation in ISO 9004 Kap. 18. 'Personal', speziell Rechnung getragen worden. Die Iso-Norm verlangt Massnahmen zur Förderung der Motivation. Weil die Motivation für ein QS-System natürlicherweise über den Menschen zu erfolgen hat, sind auch dort die primären Ursachen für Probleme und Fallstricke in der Einführung von QS-Systemen zu suchen. In diesen aus dem menschlichen Verhalten erwachsenen Widerständen lassen sich Motivationsinhibitoren eruieren. Diese stehen als Probleme, Hindernisse und Fallstricke im Wege und erschweren das Leben im QS-System insbesondere in der Einführungsphase. Auf sechs ausgewählte Themen sei hier kurz eingegangen, und es wird versucht, einige Empfehlungen zur Vermeidung dieser Stolperstellen aufzuzeigen:

\subsection{Die vorgesetzten Stellen oder auch} Sie selber stehen nur verbal aber nicht überzeugt hinter den Zielen von $Q$

- Das Management muss sich voll hinter die Qualitätskultur stellen. Diese muss aktiv vorgelebt werden. Liefern Sie persönliche Qualität, sonst tragen Sie dazu bei, das Q abzuschaffen. Die Forderung nach qualitätsbewusstem Verhalten aller Mitarbeiter wird durch das Vorbild des Chefs untermauert.

- Es soll ein Programm zur Förderung des Qualitätsbewusstseins aufgestellt werden, das z.B. periodische Auffrischungslehrgänge vorsieht. Es genügt nicht, sich mit einem einmaligen, gross aufgezogenen Schulungsprogramm zu begnuigen. Nur die geplante, permanente Weiterführung derQ-Ausbildung bringt uns dem Fernziel TQM näher.

- Informationen sollen auch bzgl. Zusammenhang und Hintergrund von Projekten objektiv an die Projektmitarbeiter weitergeben werden. Orientierungslosigkeit verunsichert und schwächt die Motivation.

- Dialogbereitschaft, insbesondere beim Austragen von Meinungsverschiedenheiten, zeugt von gegenseitiger Achtung zwischen Vorgesetzten und Mitarbeitern. Wenn wir nicht kommunizieren können, bleibt neben vielem anderem auch die Qualität auf der Strekke.

- Die Anerkennung der Arbeit und ihrer Resultate muss aktiv gelebt werden. Das Vorschlagswesen ist aufrecht zu 
erhalten. Es ist eine 'Dankekultur' zu pflegen.

\subsection{Aufwand an Zeit und Geld wird unterschätzt}

- Die Einführung von Qualitätssicherungssystemen benötigt Zeit und Ressourcen und dadurch auch erhebliche Geldmittel.Planung und Budgetierung stehen am Anfang des Aufbaus ein QS-Systems.

- Eine Terminplanung mit strukturierten Tätigkeitslisten verringert die Gefahr von Überlastungsfrust beim Mitarbeiter.

- Unnötige Hast und Zeitdruck ist zu vermeiden. Ein QS-System unter übertriebenem Zeitdruck einführen zu müssen, ist kein Q-Merkmal.

\subsection{Die Belastbarkeit der Front ist nicht unbeschränkt}

- Die Einführung von QS-Systemen erfordert den unbedingten Einbezug der Linie. Es müssen für den Zeitbedarf der Erarbeitung von Q-relevanten Unterlagen klare Prioritäten gesetzt werden. Unter Umständen müssen andere dringende Tätigkeiten des 'Daily Business' zurückgestellt werden.

- Die Akzeptanz von Resultaten aus Arbeitsgruppen und Teams ist besser als die von Einzelkämpfern. Die Normen geben die Rahmenbedingungen. Das QS-System muss vom Kollektiv der Mitarbeiter getragen werden können.

- Stäbe sind in die Vorbereitung von Arbeitspapieren und Arbeitsgrundlagen und für die Organisation der Schulung einzubeziehen. Sie dürfen auf keinen Fall als Gesetzgeber für die Linie auftreten.

\subsection{Widerstände bei der Umsetzung werden nicht analysiert}

Ein Ziel der Q-Schulung soll sein, dem Mitarbeiter klarzumachen, wo seine persönlichen Vorteile aus dem QS-System herauswachsen können.

- Mitarbeitern sollten die Vorteile einer guten Arbeitsausführung auf allen Ebenen bewusst gemacht werden. Der Mitarbeiter sieht ein, dass Qualität gut ist, wenn sich diese nicht nur auf das Produkt oder die Dienstleistung positiv auswirkt sondern auch für ihn persönlich bezahlt macht.

- Im Team müssen Werkzeuge und Hilfsmittel geschaffen werden, die dem Einzelnen in seiner persönlichen Arbeit Vorteile und Nutzen bringen. Die Erleichterung der täglichen Arbeit schafft gerade den Freiraum, den jeder Einzelne für die Entwicklung der eigenen Kreativität braucht.
- Ein akzeptiertes QS-System und das daraus resultierende Verhalten wird Erfolgserlebnisse mit sich bringen. Damit wird sich die Einführungsphase leichter durchziehen lassen. Verhalten, das zu Misserfolgen führt, wird unterlassen.

- Eine faire Behandlung aller Mitarbeiter bringt auch objektives Verhalten gegenüber dem QS-System.

Im Katalog weiterer persönlicher positiver Auswirkungen können stehen:

- Sie erringen das Vertrauen und den Respekt ihrer Mitmenschen

- Sie steigern die Möglichkeiten, interessante und herausfordernde Aufgaben zu bekommen.

- Sie verbessern Ihre Aussichten auf Beförderung.

- Sie verursachen selber weniger Fehler und vermeiden zeitaufwendige Korrekturen.

- Sie brauchen sich nicht von anderen kontrollieren zu lassen.

- Sie leisten mehr und arbeiten effizienter.

- Sie vermeiden Kritik.

- Ihr Selbstwertgefühl und Ihre Selbstachtung steigen.

- Ihr Dasein wir erfüllter und Sie erzielen mehr Lebensqualität.

\subsection{Schaffen einer nichtïberbrückba-}

ren Kluft zwischen Anspruch und

Wirklichkeit

- Klare Zielsetzungen, persönliche und projektbezogene, schaffen auch Klarheit für die Qualitätsziele. Ihnen und Ihren Mitarbeitern müssen die übergeordneten Ziele ihrer Abteilung, des Unternehmens oder der Organisation bekannt sein. Der Sinn der einzelnen Aufgaben muss klar erkannt werden können. Kenntnis der Hintergründe für die Ausführung von Aufgaben ist eine Voraussetzung für Motivation.

- Es sollten - wo möglich - quantifizierbare Massstäbe für die Qualitätserreichung aufgestellt werden. Die F+ELeitung sollte eine Leistungsanerkennung vorsehen, wenn befriedigende Qualitätsniveau erreicht werden (Incentive-System, Individuelle Leistungskomponente, F+E Preis)

- Job Description, Wichtigste Zuständigkeiten (WIZU), regeln die Tätigkeitsbereiche und die Verantwortung des einzelnen Mitarbeiters.

\subsection{In einer Firmenkultur lässt sich} nur eine $Q$-Philosophie verwirklichen

Es ist eine altbekannte und gem zitierte Tatsache: 'Der Mitarbeiter steht im Zentrum'. Dennoch wird sie oft nicht in Rechnung gestellt und übergangen. Die folgen- den Stichworte sollen zur Reflektion der Verhältnisse in Ihrer eigenen Firma anregen.

- Der Mitarbeiter muss sich wohlfühlen.

- Gegenseitiger Respekt.

- Offenheit.

- Umfassende Information; Kommunikation ermöglicht das Aufbrechen von Hierarchien und die aktive Mitgestaltung des Projektbeteiligten am Entscheidungsprozess.

- Hohe Moral.

- Vertrauen.

- Geborgenheit.

- Toleranz.

- Betonung der starken Seiten der Menschen.

- Gute Laune.

- Angemessener Zeitdruck.

Das QS-Sicherungssystem wird auch Nebeneffekte mit sich bringen, die sich auf die Qualität der Arbeitsresultate der einzelnen Organisationseinheiten auswirken werden:

- Besseres Betriebsklima

- Geringere Fluktuation

- Niedrigere Fehlzeiten

- Gemeinsame Sprache

- Kennen der gegenseitigen Anforderungen

- Weniger Ärger, Wiederholungen, Terminnot

Die grösste Herausforderung der Zukunft im Industriebereich darf nicht sein, Fabriken zu schliessen und Menschen durch die Technologie zu ersetzen. Es wird noch viel mehr darum gehen, Menschen und Technologie zu vereinigen, um in bestmöglichem Arbeitsklima bei grösstmöglicher Leistungsfähigkeit Produkte und Dienstleistungen allererster Qualität hervorzubringen. 